\title{
Untersuchungen an stromstarken Kapillarentladungen
}

\author{
Teil I. Vorbereitende Untersuchungen \\ Hans JÜrgen Kusch und Harald Schreiber \\ Institut für Experimentalphysik der Universität Kiel \\ (Z. Naturforsch. 27 a, 513-519 [1972] ; eingegangen am 28. Oktober 1971)
}

Investigations on High Current Capillary-Discharges. Part I: Preliminary Investigations

\begin{abstract}
A pulsed discharge through plastic capillaries is investigated using spectroscopic and cinematographic techniques. In the wavelength region $2600-5600 \AA$ the emission was nearly that of a black body of $30000^{\circ} \mathrm{K}$ lasting for nearly $150 \mu \mathrm{sec}$; therefore the light source can be used as a suitable radiation-standard in $\mu$ sec-spectroscopy. The optical thickness is measured and compared with calculations of continuum-intensity. Estimates of pressure and electron density lead to values of $150 \mathrm{~atm}$ max. and $2 \cdot 10^{19} \mathrm{~cm}^{-3}$ respectively. Also the plasma jet protruding from the capillary is investigated.
\end{abstract}

\section{A. Einleitung}

Die quantitativ-spektroskopische Untersuchung von Plasmen erfordert die Eichung der Empfänger Normallichtquellen bekannter Intensitätsverteilung. Die Vermessung von Hochtemperaturplasmen wird erschwert oder gar unmöglich gemacht durch die geringe Intensität herkömmlicher Strahlungsnormale wie Wolframbandlampe ${ }^{1,2}$ und Kohlebogen ${ }^{3-10}$. Während im sichtbaren Spektralbereich der Intensitätsunterschied jener Hochtemperaturplasmen verglichen mit der Strahlung des Anodenkraters eines Kohlebogens einen Faktor $10^{4}$ nicht überschreitet, beträgt dieser bei $3000 \AA$ schon $10^{5}$ und bei $2000 \AA$ bereits $10^{7}$.

Beim photographischen Meßverfahren läßt sich durch geeignete Wahl des Raumwinkelverhältnisses und durch Erweiterung des Eingangsspaltes des Spektrographen ein Intensitätsunterschied von $5 \cdot 10^{4}$ noch überbrücken; die Verwendung von Filtern bekannter Transparenz gestattet die Ausdehnung auf etwa $10^{6}$. Bei photoelektrischen Messungen ist die Kenntnis der Kennlinie des verwendeten Photomultipliers in einem viele Zehnerpotenzen überstreichenden Bereich ${ }^{11}$ notwendig. Darüber hinaus ist die photoelektrische Meßmöglichkeit geringer Strahlungsleistungen durch das Signal-Rausch-Verhältnis des Photomultipliers begrenzt.

Intensiv strahlende Hochtemperatur-Lichtquellen haben typische Emissionszeiten von einigen Mikro-

Sonderdruckanforderungen an Prof. Dr. W. Lochte-HolTGREVEN, Institut für Experimentalphysik der Universität Kiel, D-2300 Kiel, Olshausenstraße 40-60, Neue Universität, Gebäude D 6. sekunden; daher ist die Untersuchung durch stromstarke Impulse betriebener Kapillarentladungen der Gegenstand dieser Arbeit.

Für die Verwendbarkeit solcher Entladungen als Intensitätsstandard sind neben lokalisierter Emission zwecks einfacher Justierung und externer Triggerbarkeit die folgenden Forderungen zu erfüllen:

1. gute Reproduzierbarkeit der emittierten Strahlung;

2. hohe Strahlungsintensität, besonders im UV, d.h. hohe Plasmatemperatur;

3. hoher Druck zwecks Erzeugung eines linienarmen Kontinuums;

4. übersichtliche Plasmabedingungen, um auch in der Vermessung unzugänglichen Bereichen des Spektrums die Berechnung der Emission zu gestatten ${ }^{12}$.

RATH et al. ${ }^{13}$ untersuchten die Eignung stromstarker freier Hochdruckbögen als reproduzierbare UV-Lichtquelle, während MANTHEY et al. ${ }^{14}$ die Verwendbarkeit von freien als auch in Kapillaren eingeschlossenen Funken als Intensitätsstandard überprüften. ConRADS ${ }^{15-18}$ untersuchte die Eigenschaften von Impulsentladungen in evakuierten Kapillaren; doch beschränkt hier die geringe Dauer der kontinuierlichen und konstanten Emission die Anwendbarkeit. Die von Podmoshenskil und OGURTsova ${ }^{19-23}$ angegebene Kapillarlichtquelle war der Ausgangspunkt dieser Arbeit.

\section{B. Der Aufbau der Kapillarlichtquelle}

Einen Querschnitt der verwendeten Entladungslampe zeigt Abbildung 1. Zwischen zwei durch Kupferringe 
eingefaßte Kohleelektroden wurde eine durchbohrte Kunststoffplatte (Plexiglas, Polyäthylen, Teflon) von $8 \mathrm{~mm}$ Dicke eingepaßt. Die Elektroden waren mit der gleichen Durchbohrung versehen, so daß die Kapillare einen beiderseits offenen Entladungskanal bildet. Kunststoffplatte und Elektrodenhalter waren durch vier iso-

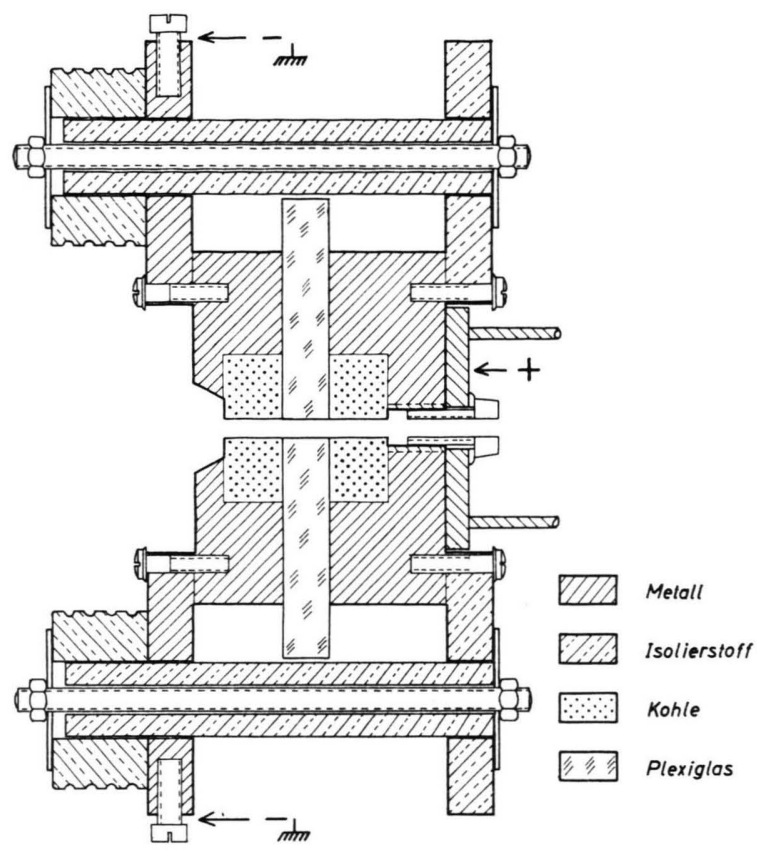

Abb. 1. Schnitt durch die Kapillarlampe.

lierte Gewindestangen zusammengepreßt. Die Kohleelektroden wurden unter dem Gesichtspunkt mechanischer Stoßfestigkeit als auch spektraler Reinheit ausgewählt; die harte Graphitkohle EK 48 (RingsdorffWerke, Mehlem/Bonn) erfüllte diese Bedingungen. Die verwendeten Kunststoffe bestehen aus Elementen mit nur wenig Linien im sichtbaren und ultravioletten Spektralbereich; daher war die Forderung 3 (Abschnitt A) leicht zu erfüllen. Als Kapillardurchmesser wurde 2 bzw. $3 \mathrm{~mm}$ gewählt; damit war neben der Ausbildung hohen Drucks und hoher Temperatur eine gute Lokalisierung der Plasmasäule erreichbar.

\section{Die Stromversorgung der Kapillar-Entladung}

Die Entladung wurde aus einem Stoßstromgenerator gespeist, der die Form einer Laufzeitkette hatte. Sechs Kondensatoren von $80 \mu \mathrm{F}$ ( $\mathrm{R} \mathrm{u} \mathrm{f} \mathrm{e} \mathrm{r} \mathrm{K.} \mathrm{G.,} \mathrm{Eckern-}$ förde) waren mit fünf Induktivitäten $L=3,6 \mu \mathrm{H}$ (11 Windungen $16 \mathrm{~mm}^{2}$ Kupferdraht auf Isolierpreßspan von $50 \mathrm{~mm}$ Durchmesser) und einer Abschlußinduktivität $L^{\prime}$ von $5,4, \mu \mathrm{H}$ mit Entladungslampe und Abschlußwiderstand verbunden. $L^{\prime}$ diente dabei gleichzeitig als Sekundärspule eines zur Zündung der Entladung notwendigen Impulstransformators (vgl. ${ }^{24}$ ). Der Abschlußwiderstand bestand aus zwei in Reihe geschalteten
Kohlestäben (Ringsdorff, RKS) von $20 \mathrm{~cm}$ Länge und $12 \mathrm{~mm}$ Durchmesser entsprechend $0,25 \Omega$ und war also dem Wellenwiderstand der Laufzeitkette $Z=(L / C)^{1 / 2}$ $=0,21 \Omega$ angepaßt. Die Dauer des rechteckförmigen Stromimpulses betrug entsprechend:

$$
\begin{gathered}
\tau=(L C)^{1 / 2} \cdot 2 n=204 \mu \mathrm{sec} \\
(n=\text { Gliederzahl der Laufzeitkette }) .
\end{gathered}
$$

Die maximale Entladestromstärke (unter Berücksichtigung der Anstiegs- und Abfallzeiten des Stromes) war $6,15 \cdot 10^{3} \mathrm{~A}$; dem entspricht bei $3 \mathrm{~mm}$ Kapillarendurchmesser eine Stromdichte von $8,7 \cdot 10^{4} \mathrm{~A} \mathrm{~cm}^{-2}$. Abbildung 2 zeigt eine schematische Darstellung der elektrischen Anordnung.

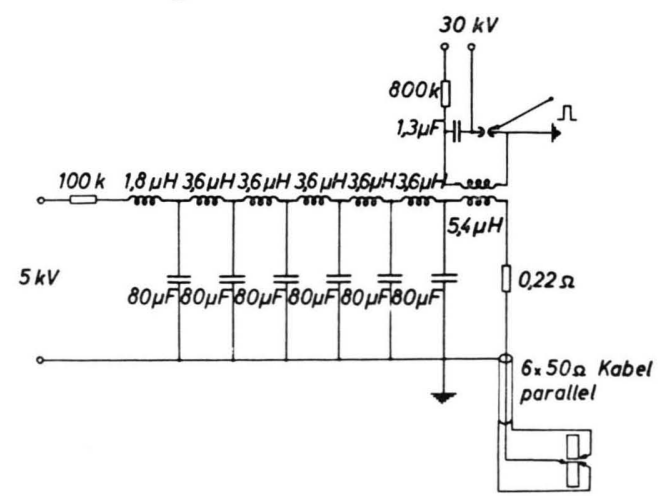

Abb. 2. Schematische Darstellung der elektrischen Anordnung.

\section{Die Entladungserscheinung}

Bei den umgesetzten Leistungen ist die Kapillarentladung von einem stürmischen Verdampfen des Wandmaterials begleitet. Darauf weist die Vergrößerung des Kapillardurchmessers von Entladung zu Entladung als auch das Auftreten von C I-, C II- und HLinien in den Plasmastrahlen hin, die beiderseits aus der Kapillare austreten.

Die Verwendung enger Kapillaren und hoher Stromstärken führt also zu einer größeren Belastung des Wandmaterials; im Hinblick auf die Reproduzierbarkeit der Strahlung bei mehrfacher Verwendung derselben Kapillare dürfen daher nicht zu enge Kapillaren und nicht zu hohe Stromstärken verwendet werden.

\section{E. Die zeitliche Entwicklung des Plasmastrahls}

Zur Gewinnung eines Überblicks über Dauer und Intensität des Leuchtens aus dem Plasmastrahl und dessen Einfluß auf die Ausstrahlung der Kapillare wurden Schmieraufnahmen gemacht.

Eine Linse $\mathrm{L}_{1}$ bildete den Plasmastrahl verkleinert auf einen längs der Strahlrichtung orientierten Spalt ab, der die Mittelachse des Plasmastrahls ausblendete. Ein Objektiv $\mathrm{L}_{2}$ bildete den beleuchteten Spalt sodann auf den rotierenden Film einer Trommelkamera (Strobodrum, Impulsphysik F. Früngel, Hamburg) ab. 
Die Plasmazufuhr aus der Kapillare in den Plasmastrahl ist nach Ende des Stromimpulses (ca. $200 \mu \mathrm{sec}$ ) beendet; danach leuchten nur noch die entfernteren Zonen des Strahls. Für die Frontgeschwindigkeit ergab sich ein mittlerer Wert von $7 \cdot 10^{4} \mathrm{~cm} / \mathrm{sec}$, während aus den Bahnen mitbewegter Kohlepartikel (vgl. ${ }^{25}$ ) als unterer Wert für die Strömungsgeschwindigkeit im Plasmastrahl $9 \cdot 10^{5} \mathrm{~cm} / \mathrm{sec}$ folgt.

\section{F. Die Bestimmung der Plasmadaten der Kapillar-Entladung}

\section{Spektroskopische Messungen}

\section{a) Der optische Aufbau}

Für die quantitativ-spektroskopischen Untersuchungen wurde die photographische Methode gewählt. Eine Quarzlinse bildete die Kapillarmitte in Richtung der Achse über einen Oberflächenspiegel etwa vierfach vergrößert auf den Spektrographenspalt ab. Durch Wahl der Größe der begrenzenden Blende gelangte nur die Strahlung achsennaher Zonen zur Beobachtung. Verwendet wurde ein Quarzspektrograph Q 24 (VEB Jena); seine Lineardispersion variierte von $5,5 \AA / \mathrm{mm}$ bei $2200 \AA$ bis zu $67 \AA / \mathrm{mm}$ bei $5200 \AA$, die lichtquellenseitige Öffnung betrug $1: 12,5$. Als Normalstrahler diente ein Kohlebogen ${ }^{8}$, dessen positiver Krater über den Spiegel vergrößert auf den Spalt des Spektrographen abgebildet wurde. Eine Spaltblende begrenzte in beiden Fällen die Höhe des Spektrums auf $5 \mathrm{~mm}$. Zur Einstellung der Belichtungszeit von $120 \mu$ sec diente eine vor dem Spektrographenspalt angeordnete Sektorscheibe, die über Photodiode und Hilfslichtquelle den Lichtimpuls mit einer Verzögerung von $70 \mu$ sec gegenüber dem Einsetzen der Entladung herausschnitt. Bei der Belichtung des Normalstrahlerspektrums wurde zusätzlich ein Zentralverschluß (1/50 sec) verwendet; die Kontrolle, daß nur ein Impuls in den Spektrographen eintrat, erfolgte über einen kleinen zwischen Spalt und Sektorscheibe angebrachten Spiegel in Verbindung mit einer photoelektrischen Meßanordnung. Die Schwärzungsmarken wurden mit einem Platinstufenfilter hergestellt, dessen Transmission als Funktion der Wellenlänge zuvor photoelektrisch bestimmt war. Als Hintergrundslichtquelle hierfür diente eine mit der gleichen Stromversorgung betriebene Argon-Hochdrucklampe, wie sie schon in ${ }^{13}$ und ${ }^{24}$ beschrieben wurde. Mit Hilfe zweier Quarz-Lithiumfluorid-Achromaten wurde das Stufenfilter gleichmäßig ausgeleuchtet. Zur Überstreichung eines großen Intensitätsbereichs erhielt jede Platte zwei Intensitätsmarken mit überdeckender Schwärzung (durch Variation des Eintrittsspalts). Als Aufnahmematerial verwendeten wir Ilford-R 10-Platten; entwickelt wurde in Microphen bei $20^{\circ} \mathrm{C} 12 \mathrm{~min}$. Selbst bei voller Breite des Spektrographenspaltes und größtmöglichem Raumwinkel konnte die Intensitätsmessung der Kapillarlampe wegen mangelnder Normalstrahlerhelligkeit nur bis $3000 \AA$ durchgeführt werden; zur Ausdehnung bis $2600 \AA$ diente das folgende Verfahren: die Platte wurde jeweils zehnmal mit Licht- impulsen der Kapillarlichtquelle und der Stufenanordnung sowie des Normalstrahlers belichtet, erstere jedoch abgeschwächt durch ein Platinrhodium-Graufilter (Balzers, Liechtenstein) mit photoelektrisch als Funktion der Wellenlänge vermessener Transparenz (ca. $10 \%)$.

\section{b) Die Ergebnisse der spektroskopischen Messungen}

Bei Voruntersuchungen über die Abhängigkeit der Kapillarstrahlung vom Wandmaterial erwiesen sich Polyäthylen und Teflon als weniger geeignet; alle weiteren Messungen wurden daher mit Plexiglas (Polymetacrylester) ausgeführt. Abbildung 3 zeigt die Intensität in Abhängigkeit von Ladespannung und Kapillardurchmesser mit der Wellenlänge als Parameter.

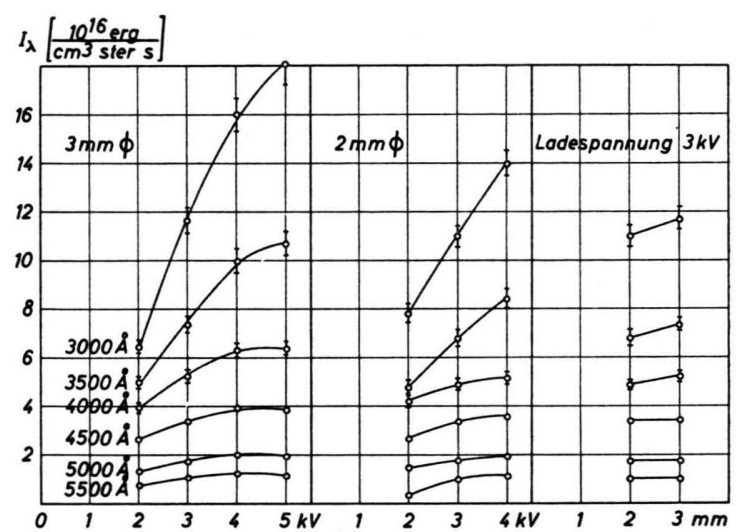

Abb. 3. Die Intensität als Funktion von Kapillardurchmesser und Ladespannung.

Diese Darstellung ermöglicht in Verbindung mit Messungen der Verdampfungsrate Schlüsse auf die Vorgänge im Inneren der Kapillare. Erhöhte Leistungszufuhr und Verringerung des Kapillardurchmessers führen zu starker Erhöhung der Verdampfungsrate und zu steilem Druckanstieg, jedoch zu keiner nennenswerten Temperaturerhöhung.

a) Reproduzierbarkeit. Zur Prüfung der Reproduzierbarkeit der ausgesandten Strahlung wurde sowohl eine Vielzahl von Messungen unter gleichen Entladungsbedingungen als auch die Strahlungsintensität mehrerer Entladungen durch dieselbe Kapillare als Funktion der Wellenlänge untersucht. Die Spektren wurden auf derselben Platte aufgenommen, um den photographischphotometrischen Fehler zu unterdrücken; die Schwankungen der Strahlungsintensität variierten von ca. $1 \%$ im Sichtbaren bis ca. 5\% im UV.

$\beta)$ Intensitätsverteilung. Abbildung 4 zeigt die Ergebnisse der spektroskopischen Absolutmessungen und im Vergleich dazu Kirchhoff-Planck-Isothermen von 26000,28000 und $30000{ }^{\circ} \mathrm{K}$. Der Messung liegen drei Spektren zugrunde; die Meßpunkte sind Mittelwerte, die Fehlergrenzen wurden aus den Abweichungen von diesen ermittelt.

y) Die Ermittlung der optischen Dicke. Die optische Dicke des Kapillarplasmas wurde nach einem allgemein 


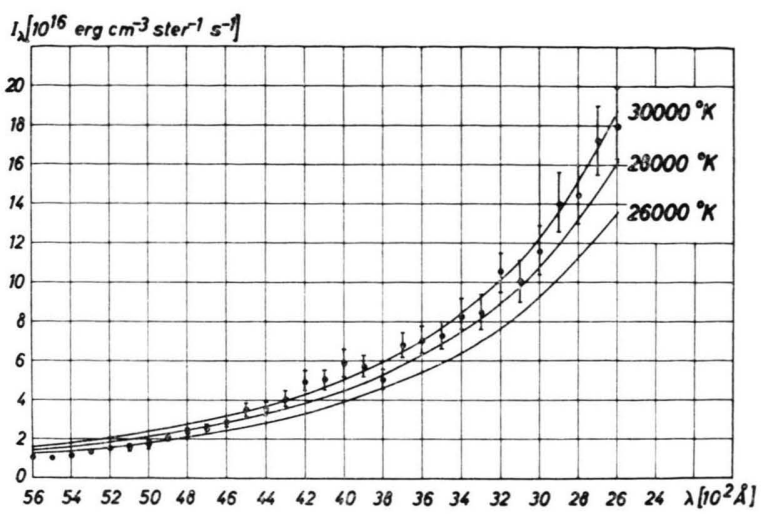

Abb. 4. Die spektrale Strahldichteverteilung der Kapillarlampe im Vergleich zu den Kirchhoff-Planck-Isothermen von 26000 , 28000 und $30000^{\circ} \mathrm{K}$.

bekannten Verfahren bestimmt. Hinter der dem Spektrographen abgewandten Öffnung der Kapillare wurde ein kleiner Hohlspiegel $(f=4 \mathrm{~cm})$ im Abstande $2 f$ von der Kapillarmitte aufgestellt, der das austretende Strahlenbündel in sich reflektierte. Das mittels der Sektorscheibe (vgl. Abschn. F, l a) festgelegte Belichtungsintervall lag dabei zeitlich jeweils vor der Ankunft des Plasmastrahls auf der Spiegeloberfläche und vor deren Zerstörung. Die Verdopplung der optischen Schichtlänge führt im Falle optisch dicker Schicht zu keiner Intensitätssteigerung; Abb. 5 zeigt die $\mathrm{Meß}$ ergebnisse für zwei Kapillardurchmesser.

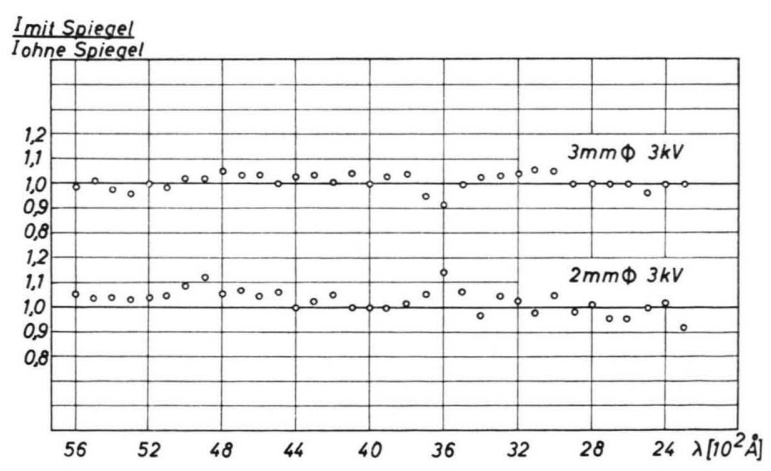

Abb. 5. Zur optischen Dicke des Kapillarplasmas.

d) Die Isotropie der emittierten Strahlung. Radiale Teilchendichte- und Temperaturgradienten ändern die optischen Eigenschaften der zur Emission beitragenden Zonen, bestimmen also wesentlich die für quantitativspektroskopische Anwendungen notwendige Isotropie der Ausstrahlung. Die Untersuchungen ergaben im Bereich bis zu $2,5 \cdot 10^{-2}$ ster Proportionalität zwischen Strahlungsstrom und dem der Kapillare zugewandten Raumwinkel innerhalb Fehlergrenzen von 5\%.

ع) Spektroskopische Untersuchungen des Plasmastrahls. In verschiedenen Abständen von der Austrittsöffnung wurden side-on-Spektren des Plasmastrahls aufgenommen; die Belichtungszeit betrug wieder $120 \mu \mathrm{sec}$.
Die Verzögerungszeit zwischen dem Einsetzen der Entladung und der Belichtung war so eingestellt, daß die Sektorscheibe den Eintrittsspalt des Spektrographen erst freigab, nachdem der Plasmastrahl gerade den Untersuchungsort erreicht hatte: durch diese Methode wurde die Strahlung eines Volumelements zu verschiedenen Zeitpunkten registriert. Im Gegensatz zu dem von einigen Absorptionslinien der Elemente des Elektrodenmaterials unterbrochenen kontinuierlichen end-onSpektrum zeigt das Spektrum des Plasmastrahls die Linien der Elemente des Kapillar- und des Elektrodenmaterials in Emission. Die zusätzlich auftretenden CNBanden haben ihren Ursprung in turbulenter Durchmischung der Randzonen des Strahls mit der umgebenden Luft.

Radiale Verteilungen der Elektronendichte wurden nach Abel-Reduktion ${ }^{26}$ aus dem Linienprofil von $\mathrm{H}_{\beta}$ nach GRIEM ${ }^{27}$ erhalten. Die Elektronendichte ist vom Radius praktisch unabhängig und nimmt mit zunehmender Entfernung von der Austrittsöffnung langsam $\mathrm{ab}$; die Werte liegen um $2 \cdot 10^{17} \mathrm{~cm}^{-3}$.

Zur Bestimmung der radialen Temperaturverteilung im Plasmastrahl wurden die radialen Intensitäten der Ca II-Linien $3933,7 \AA\left(4,{ }^{2} \mathrm{~S}-4{ }^{2} \mathrm{P}^{0}\right)$ und $3179,3 \AA$ $\left(4^{2} \mathrm{P}^{0}-4{ }^{2} \mathrm{D}\right)$ verglichen. Mit den nach der Methode der Coulomb-Approximation berechneten Übergangswahrscheinlichkeiten ${ }^{28}$ ergeben sich Temperaturen um $30000{ }^{\circ} \mathrm{K}$ mit Fehlern von ca. $30 \%$; diese Werte weichen also kaum von denen innerhalb der Kapillare ab.

Der kontinuierliche Absorptionskoeffizient im Plasmastrahl wurde aus Messungen der Radialverteilung des schwachen kontinuierlichen Untergrundes gewonnen. Bezogen auf die Intensität eines schwarzen Körpers von $30000^{\circ} \mathrm{K}$ erhält man dann bei $4600 \AA$ bzw. $3400 \AA$ Werte von $8 \cdot 10^{-3} \mathrm{~cm}^{-1}$ bzw. $9 \cdot 10^{-3} \mathrm{~cm}^{-1}$. Bei einer absorbierenden Schichtlänge von $15 \mathrm{~cm}$ ergibt sich damit im Bereich $\lambda<4600 \AA$ eine Absorption der end-onStrahlung der Kapillare von weniger als 1\%. Damit ist der Einfluß des Plasmastrahls auf die von der Kapillare emittierte Strahlung vollständig zu vernachlässigen.

\section{Die Bestimmung des Drucks in der Entladung}

Da bei Kenntnis von Temperatur und Druck die Plasmakomponenten aus der chemischen Zusammensetzung $\left(\mathrm{C}_{5} \mathrm{H}_{8} \mathrm{O}_{2}\right)$ des Wandmaterials berechnet werden können, ist die Messung des Plasmadrucks von wesentlicher Bedeutung für das Verständnis der Entladung: optische und elektrische Eigenschaften des Plasmas sind dann berechenbar. Zur Messung des Drucks diente eine ballistische Methode. Ein speziell angefertigter Kupferpfropfen wurde mittels Vakuumfett gut dichtend in die vordere Kohleelektrode eingesetzt, die hintere Elektrodenbohrung jedoch verschlossen. Der bei der Entladung in der Kapillare entstehende Druck preßt den Pfropfen heraus; dieser wird sodann von einem an zwei Fäden aufgehängten Pendelkörper aufgefangen, dessen Auslen- 
kung gemessen wird. Unter der Voraussetzung der Verdoppelung des Plasmadrucks beim Verschließen der hinteren Elektrodenöffnung folgt dann aus Energie- und Impulssatz:

$$
P=\frac{M+m}{2 \pi r^{2} \Delta t}(g l)^{1 / 2} \frac{s}{l} ;
$$

hierin ist $P$ der Druck, $M$ bzw. $m$ die Masse von Pendelkörper bzw. Kupferpfropfen, $l$ der Abstand Aufhängepunkt - Schwerpunkt des Pendelkörpers, $s$ die Auslenkung und $\Delta t$ die Zeitdauer der Plasmaproduktion. Da der Einfluß der geänderten Versuchsbedingungen auf den Druck nur schwer zu übersehen ist, kommt der ballistischen Druckmessung trotz der gut reproduzierbaren Ergebnisse nur begrenzte Genauigkeit zu. (Über eine Druckmessung unter Verwendung piezoelektrischer Sonden wird später im Teil II berichtet.) In Kapillaren von $3 \mathrm{~mm}$ Durchmesser erreicht der Druck bei einer Ladespannung von $3 \mathrm{kV} 150 \mathrm{~atm}$.

\section{G. Die Berechnung der Plasmazusammensetzung und der Strahlungseigenschaften eines Plexiglas-Plasmas}

Da Temperatur und Druck im Plasma der Kapillarentladung hinreichend genau gemessen wurden, kann die Plasmazusammensetzung als Funktion dieser Größen berechnet werden. Die Kenntnis der Teilchendichten gestattet dann eine Berechnung der optischen und elektrischen Eigenschaften des Plasmas. Wie schon erwähnt, wird bei den vorliegenden Entladungsbedingungen das Plasma der Kapillare allein durch die chemische Zusammensetzung des die Kapillare bildenden Wandmaterials bestimmt (in unserem Falle also $\mathrm{C}_{5} \mathrm{H}_{8} \mathrm{O}_{2}$ ).

\section{Die Berechnung der Teilchendichten}

Zur Berechnung der Teilchendichten wurde das System der Saha-Gleichungen, der Quasineutralitätsbeziehung und der stöchiometrischen Verhältnisse iterativ mit einem Rechenprogramm von MEwEs ${ }^{29}$ gelöst; die Berücksichtigung der Plasmakorrekturen erfolgte nach GRIEM ${ }^{30}$. Abbildung 6 zeigt das Ergebnis dieser Rechnungen für einen Druck von $150 \mathrm{~atm}$. Berechnungen für niedrigere Drucke zeigen gute Übereinstimmung zwischen der im Plasmastrahl gemessenen Elektronendichte und dem Umgebungsdruck von ca. $1 \mathrm{~atm}$; die Länge der das

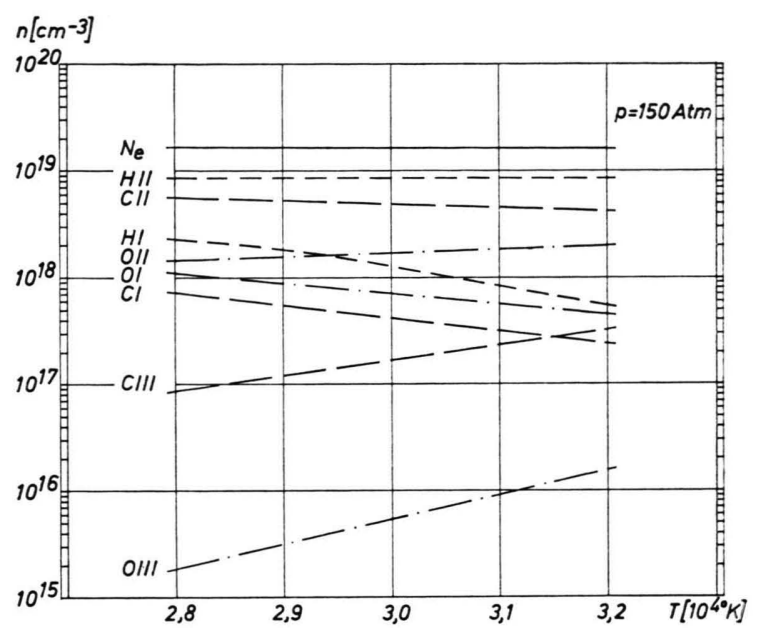

Abb. 6. Die Plasmazusammensetzung des Plexiglasplasmas als Funktion der Temperatur für den Druck $150 \mathrm{~atm}$.

Kontinuum aussendenden Plasmasäule ist also streng begrenzt und entspricht der Länge der Kapillare (einschließlich der Elektrodenbereiche).

\section{Die optische Intensität}

Die Strahlungsintensität einer homogenen Plasmasäule der Länge $l$ ist nach der Gleichung

$$
I_{\lambda}=B_{\lambda}\left(1-\exp \left\{-\varkappa_{\lambda} l\right\}\right)
$$

berechenbar, sofern der Absorptionskoeffizient $\varkappa_{\lambda}(\lambda, T)$ bekannt ist. Dieser setzt sich zusammen aus den gebunden-frei-Übergängen der verschiedenen Plasmakomponenten und dem frei-frei-Kontinuum der Elektronen.

Nach SCHLÜTER ${ }^{31}$ gilt für den auf ein Atom bezogenen gebunden-frei-Anteil im Anschluß an KRAMERS ${ }^{32}$ und UNSÖLD ${ }^{33}$ :

$$
\begin{gathered}
\sigma(\lambda, T)=\frac{32 \pi^{2} e^{6}}{3^{3 / 2} h^{4} c^{4}} \frac{k T}{u(T)} \lambda^{3} \exp \left(-\frac{h c}{\lambda_{1} k T}\right) \\
\cdot \exp \left(\frac{h c}{\lambda k T}-1\right) \gamma \cdot \xi(\lambda, T)
\end{gathered}
$$

mit $u(T)$ Zustandssumme des betreffenden Elements, $\lambda_{1}$ Hauptseriengrenze für Wasserstoff $(=911,8 \AA$ ), $\gamma$ das Verhältnis der statistischen Gewichte des Grundzustands des Elternions des betrachteten Elemenst zum Elternion des Wasserstoffs.

Der Faktor $\xi(\lambda, T)$ beschreibt die Abweichung des quantenmechanisch berechneten Absorptionsquerschnitts vom klassischen wasserstoffähnlichen Wert. 


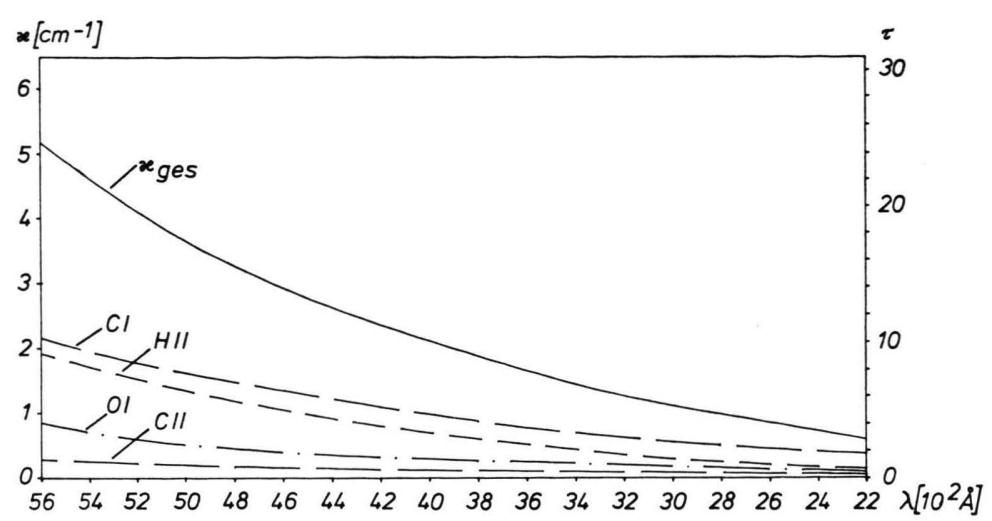

Abb. 7. Der spektrale Verlauf des gesamten Absorptionskoeffizienten und der optischen Tiefe. Als Schichtlänge für die Berechnung der optischen Tiefe wurde die Kapillarlänge einschl. der Elektrodenbohrungen benutzt.

Den Absorptionskoeffizienten in der bekannten Dimension $\mathrm{cm}^{-1}$ erhält man dann gemäß :

$$
\varkappa(\lambda, T)=\sum_{i} N_{i} \sigma_{i}
$$

nach Summation über alle Teilchensorten.

Die Beiträge von C I, C II und O I wurden den Tabellen von PEACH ${ }^{34}$ entnommen, der Anteil der Elektronen und Wasserstoffionen nach KRAMERSUNsöLD ${ }^{35}$ berücksichtigt. Für die Teilchensorten H I, O II, O III und C III mit $\xi=1$ berechnete Beiträge waren vernachlässigbar. Abbildung 7 zeigt das spektrale Verhalten des gesamten Absorptionskoeffizienten sowie den Verhauf der optischen Dicke des Kapillarplasmas. Das Plasma der Kapillare strahlt also im gesamten vermessenen Bereich wie ein schwarzer Körper der Plasmatemperatur.

\section{Elektrische Leitfähigkeit und Leistungsumsatz}

Nach ${ }^{36}$ berechnet sich die elektrische Leitfähigkeit eines Plasmas nach der Gleichung:

$$
\sigma=\frac{e^{2} \pi^{1 / 2}}{\left(8 m_{\mathrm{e}}\right)^{1 / 2}} \frac{n_{\mathrm{e}}}{(k T)^{1 / 2}} \frac{1}{\sum_{j} n_{j} Q_{\mathrm{e} j}} ;
$$

$Q_{\mathrm{e} j}$ sind die Querschnitte der von den Elektronen gestoßenen Partner. Mit

$$
\begin{array}{ll}
Q_{\mathrm{eH}}=(20 \pm 3) \cdot a_{0}^{2} & \left(\text { siehe }^{37-42}\right), \\
Q_{\mathrm{eC}}=(25 \pm 6) \cdot a_{0}{ }^{2} & \left(\text { siehe }^{43}\right), \\
Q_{\mathrm{eO}}=(22 \pm 7) \cdot a_{0}{ }^{2} & \text { siehe } \left.^{43}\right),
\end{array}
$$

sowie mit

$$
Q_{\mathrm{e} i}=\frac{0,5677^{2}}{\gamma_{\mathrm{E}}} \frac{e^{4}}{(k T)^{2}} \ln \frac{k T}{Z^{2} e n_{\mathrm{e}}^{1 / 3}}
$$

$\gamma_{\mathrm{E}}(Z=1)=0,5816$ und $\gamma_{\mathrm{E}}(Z=2)=0,683$ (s. $\left.{ }^{36}\right)$ folgt letztlich für einen Druck von $150 \mathrm{~atm}$ :

$$
\sigma=29,0 \cdot 10^{13} \mathrm{sec}^{-1} \text {. }
$$

Die in der Kapillare umgesetzte elektrische Leistung ergibt sich zu

$$
L=1,1 \cdot 10^{6} \mathrm{Watt} ;
$$

diese wird wesentlich zur Verdampfung des Wandmaterials und seiner Dissoziation und Ionisation benutzt sowie in Strömungsenergie umgesetzt.

\section{H. Schlußbemerkung}

In dieser Arbeit wurde über kinematographische und spektroskopische Untersuchungen an Kapillarentladungen durch Kunststoffe, besonders Plexiglas, berichtet. Dabei führten die optischen Messungen letztlich zur Entwicklung eines HochtemperaturStrahlungsnormals für die Kurzzeitspektroskopie. Druckmessungen in Zusammenhang mit Berechnungen der Plasmazusammensetzung gestatten die $\mathrm{Ab}$ schätzung der optischen Dicke aus der Kontinuumsintensität.

In einem späteren Teil II der Untersuchungen an Kapillarentladungen wird über kinematische und elektrische Messungen sowie über piezoelektrische Druckmessungen berichtet werden. Teil III enthält dann photoelektrische Präzisionsmessungen der Strahlungsintensität bis zu $2100 \AA$.

Die Verfasser danken dem Direktor des Instituts für Experimentalphysik der Universität Kiel, Herrn Prof. Dr. W. Lochte-HoltGreven, für die vielfältige Unterstützung bei der Anfertigung dieser Arbeit. Der Bundesminister für Bildung und Wissenschaft trug durch die Bereitstellung von Sachmitteln zum Gelingen der Untersuchungen bei. 
1 J. C. DE Vos, Physica 20, 690 [1954].

2 R. E. Larrabee, J. Opt. Soc. Ainer. 49, 619 [1959].

3 H. G. McPherson, J. Opt. Soc. Amer. 30, 189 [1940].

4 J. Euler, Ann. Phys. 11, 203 [1953].

5 J. Euler, Ann. Phys. 14, 145 [1954].

6 M. R. Null u. W. W. Lozier, J. Opt. Soc. Amer. 52, 1156 [1962].

7 J. P. Mehltretter, Dissertation, Heidelberg 1962.

8 H. Magdeburg, Z. Naturforsch. 20 a, 980 [1965]. - H. Magdeburg u. U. Schley, Z. Angew. Phys. 20, 465 [1966].

9 A. T. Hattenburg, Appl. Optics 6, 95 [1967].

10 K. Schurer, Appl. Optics 7, 461 [1968]; Thesis, Utrecht 1968.

11 F. KaRSTENSEN u. H. J. KusCH, Z. Naturforsch. 24 a, 1283 [1969].

12 P. Bogen, H. Conrads u. D. Rusbüldt, Z. Phys. 186, 240 [1965].

13 J. Rath, H. Manthey u. W. Bötticher, Z. Angew. Phys. 22, 350 [1967].

14 H. Manthey u. W. Bötticher, Z. Angew. Phys. 25, 77 [1968].

15 H. Conrads, Berichte der KFA Jülich Nr. 88 [1962].

16 H. Conrads u. H. HarTwig, Berichte der KFA Jülich Nr. 123 [1963].

17 H. Conrads, Berichte der KFA Jülich Nr. 300 [1966].

18 H. Conrads, Z. Phys. 200, 444 [1967].

19 N. N. Ogurtsova u. J. V. Podmoshenskir, Opt. Spectrosc. 4, 539 [1958].

20 N. N. Ogurtsova, J. V. Podmoshenskil u. M. J. Demidov, Optico Mekhan. Prom. 1, 1 [1960].

21 N. N. Ogurtsova u. J. V. Podmoshenskir, Fiz. Problemy Spektroskopii 1, 129 [1962].

22 N. N. Ogurtsova, J. V. Podmoshenskin u. V. M. SheleMINA, Opt. Spectroscopy 15, 404 [1963].
23 N. N. Ogurtsova, J. V. Podmoshenskil u. V. M. SheleMINA, Opt. Spectroscopy 16, 514, [1964].

24 H. J. KUSCH, Z. Astrophys. 67, 64 [1967].

25 W. Hagenah, Z. Phys. 128, 279 [1950].

26 U. Sörensen u. J. Richter, Koeffizientenschema zur Lösung der Abelschen Integralgleichung, Laborbericht, Kiel 1961.

27 H. R. Griem, A. C. Kolb u. K. Y. Shen, Phys. Rev. 116, 4 [1959] ; vgl. H. R. GrIeM, Plasma Spectroscopy, McGrawHill Co., London 1964.

28 H. R. GRIEM, NRL-Report 6085, 16 [1964] ; vgl. H. R. GrIeM, Plasma Spectroscopy, McGraw-Hill Co., London 1964.

29 E. R. MEwEs, Diplomarbeit, Kiel 1966.

30 H. R. GRIEM, Phys. Rev. 128, 997 [1962].

31 D. SCHLÜTER, Dissertation, Kiel 1964; vgl. D. SCHLÜTER, Z. Astrophys. 61, 67 [1965].

32 H. A. Kramers, Phil. Mag. 46, 836 [1923].

33 A. Unsöld, Ann. Phys. 33, 607 [1938].

34 G. PEACH, Memoirs of the Roy. Astronom. Soc. 73, Part 1 [1970].

35 W. Finkelnburg u. Th. Peters, Handbuch der Physik, Bd. 28, Springer-Verlag, Berlin 1957.

36 W. Finkelnburg u. H. Maecker, Handbuch der Physik, Bd. 22, Springer-Verlag, Berlin 1956.

37 R. T. Brackmann, W. L. Fite u. R. H. Neynaber, Phys. Rev. 112, 1157 [1958].

38 B. H. Bransden, A. Dalgarno, T. L. John u. M. J. SeaTON, Proc. Roy. Soc. London A 71, 877 [1958].

39 R. P. McEachran u. P. A. Fraser, Canad. J. Phys. 38, 317 [1960].

40 K. Smith, Phys. Rev. 120, 845 [1960].

41 T. L. JoHn, Proc. Phys. Soc. London 76, 532 [1960].

42 S. Geltmann, Phys. Rev. 119, 1283 [1960].

43 H. Maecker, Ann. Phys. 18, 441 [1956]. 